\title{
INSAR PERFORMANCE FOR LARGE-SCALE DEFORMATION MEASUREMENT: IMPACT OF TROPOSPHERIC CORRECTIONS AND VALIDATIONS
}

\author{
Alessandro Parizzi, Ramon Brcic, Francesco De Zan \\ Remote Sensing Technology Institute German Aerospace Center (DLR) \\ Münchenerstraße 2082234 Weßling; alessandro.parizzi@dlr.de
}

\begin{abstract}
This paper deals with the analysis of InSAR performance for large-scale deformation measurement. The study evaluates the use of models, especially numerical weather prediction reanalysis, to mitigate disturbances in SAR interferograms. The impact of such corrections is evaluated and, using GNSS measurements, the predicted error bars are validated on a large Sentinel-1 dataset.
\end{abstract}

Index Terms - InSAR, performance, deformation, numerical weather prediction (NWP), GNSS

\section{A MODEL FOR TROPOSHERE DRIVEN INTERFEROMETRIC PERFORMANCE}

The use of NWP for mitigating the tropospheric signal in the interferometric phase is limited both in accuracy, due to the limitations of the model itself, and in resolution. The best sampling available is $9 \mathrm{~km}$ in the case of ECMWF operational products, several orders of magnitude larger than the SAR resolution, even after interferometric multi-looking. The accuracy of NWP can be strongly site dependent since it depends on data availability and the fit of the model to actual tropospheric conditions. For the ECMWF products considered here, there is a strong dependence of accuracy on latitude [1]. However, it should be pointed out that since the interferograms are intrinsically relative, the impact of model bias in the simulated interferometric phase is limited. More important is the variance of the error between the real zenith delay $z_{t}$ and the modeled delay $\widehat{z}_{t} \epsilon=z_{t}-\widehat{z}_{t}$. This can be seen as a Gaussian process in time and space with mean $\mu_{\epsilon}$, variance $\sigma_{\epsilon}^{2}$ and spatial correlation function $\Lambda$. The interferometric error between two points $A$ and $B$ for reference acquisition $r$ and secondary acquisition $s$ is

$$
\phi_{\epsilon}=\left(\epsilon_{r, A}-\epsilon_{s, A}\right) \frac{e^{-\frac{h_{A}}{H}}}{\cos \theta_{A}}-\left(\epsilon_{r, B}-\epsilon_{s, B}\right) \frac{e^{-\frac{h_{B}}{H}}}{\cos \theta_{B}}
$$

where $\theta$ is the local incidence angle. The effect of the altered path delay due to topography is taken in account using the exponential mapping function as in [2] with $h_{A}$ and
$h_{B}$ the height of the points w.r.t to the ellipsoid and $H$, the so called height of the atmosphere, typically fixed to $7 \mathrm{~km}$. Assuming the error is first-order stationary, the mean interferometric phase error is $\mathbb{E}\left[\phi_{\epsilon}\right]=0$ even if the residual error has non-zero mean, $\mu_{\epsilon} \neq 0$ as visible from Equation 1. Assuming the atmosphere and the error are temporally uncorrelated, the interferometric phase error power is

$$
\begin{gathered}
\mathbb{E}\left[\phi_{\epsilon}^{2}\right]=2 \sigma_{\epsilon}^{2} \frac{e^{-\frac{2 h_{B}}{H}} \cos ^{2} \theta_{A}+e^{-\frac{2 h_{A}}{H}} \cos ^{2} \theta_{B}}{\cos ^{2} \theta_{A} \cos ^{2} \theta_{B}}- \\
-\frac{4 \Lambda(A, B)}{\cos \theta_{A} \cos \theta_{B}} e^{-\frac{h_{B}+h_{A}}{H}}
\end{gathered}
$$

$\Lambda$ is a correlation function that $\Lambda(A \equiv B)=\sigma_{\epsilon}^{2}$ and $\Lambda(A, B) \rightarrow 0$ at large distance. The residual error in the interferometric phase between points $A$ and $B$ described in Equation 2 is a parametrization of the sample variograms that can be calculated from data. Equation 2 approaches 0 as the distance $d \rightarrow 0$ and is $\propto \sigma_{\epsilon}^{2}$ as $d \rightarrow \infty$. That means that at large distances $(\geq 40-50 \mathrm{~km})$ the interferometric measurements become limited by the accuracy of the models used for the correction.

In order to study the troposphere induced error and its mitigation through the use of NWP, short time interferograms are generated from every acquisition with the next. This approach should prevent bias due to a common reference as well as eventual seasonality present in the tropospheric delay and the impact of deformation. The latter point requires clarification. The fast revisit time of the Sentinel-1 mission allows temporal baselines of 6,12 or in worse cases, 24 days. Since the residual error, including effects such as tropospheric turbulence, are assessed to be on the order of centimeters, deformation rates of several tens of $\mathrm{cm} / \mathrm{y}$ would be necessary in order to produce effects comparable to those from the troposphere.

The tropospheric error is considered temporally uncorrelated. This hypothesis should hold at least for the troposphere corrected phase since all seasonal effects are included in the NWP and are hence compensated. The interferometric phase variogram between acquisitions $n$ and $m, \Gamma_{n, m}$, is then the sum of the two variograms of the errors in acquisition $n$ and 


$$
\begin{aligned}
& m, \Gamma_{n} \text { and } \Gamma_{m} \\
& \begin{aligned}
\Gamma_{n, m}(d) & =\mathbb{E}\left[\left(\left(\phi_{n}(A)-\phi_{n}(B)\right)-\left(\phi_{m}(A)-\phi_{m}(B)\right)\right)^{2}\right] \\
& =\Gamma_{n}+\Gamma_{m}
\end{aligned}
\end{aligned}
$$

where $\phi$ is the phase at points $A$ and $B$ and $d$ is the distance between the points.By averaging the set of short time baseline variograms $\bar{\Gamma}=\mathbb{E}[\Gamma]$, it is possible to derive the average behavior of the residual interferometric phase error. Performing this experiment before and after the NWP correction allows an analysis of the impact of the corrections at different scales. The interferometric data and NWP models have different resolutions, hence different behavior is expected at different scales. This is particularly important in tectonic strain applications where high relative accuracy is required at large scales. Assuming the troposphere is the main source of error at large distances ( $\geq 40-50 \mathrm{~km})$, one can now derive its impact on deformation rate measurements [3]. The variogram of the linear deformation rate estimates is obtained by scaling $\bar{\Gamma}$ by the linear regression formula [4],

$$
\Gamma_{\mathrm{v}}(d)=\frac{1}{2} \frac{\lambda^{2}}{16 \pi^{2}} \frac{\bar{\Gamma}(d)}{M \sigma_{t}^{2}}
$$

where the factor $1 / 2$ accounts for the common reference acquisition, $M$ is the number of acquisitions and $\sigma_{t}^{2}$ is the spread of the temporal sampling $\sigma_{t}^{2}=\sum_{i} t_{i}^{2} / M-$ $\left(\sum_{i} t_{i} / M\right)^{2}$

\section{IMPACT OF THE TROPOSPHERIC CORRECTIONS}

Variograms are a convenient way to characterize the tropospheric component of the deformation rate precision. By comparing the interferometric phase variograms before and after the correction, it should be possible to observe the gain at different scales. Since the data used for compensation are not provided at the same resolution of the interferograms, a significant reduction of the tropospheric contribution can only be expected at scales larger than the NWP model resolution. Here, ECMWF ERA-5 data are used with a spatial gridding of $30 \mathrm{~km}$. It should be noted that the accuracy of the correction has been demonstrated to vary considerably worldwide [1].

In order to comprehensively evaluate correction performance, 146 Sentinel-1 stacks have been processed over various regions of the world. Each of these stacks spans at least 4 years and contains up to 200 acquisitions. As described in Section 1, only short time baseline interferograms were used to ensure the troposphere signal dominates.

An evaluation of the performance at large distances was also performed. The average variograms before and after corrections were fitted using typical variogram models (exponential, Gaussian and spherical) hence reducing the variance of the variogram estimate. Using this information together with temporal sampling information and Equation 4, the performance in measuring the deformation rates at $150 \mathrm{~km}$ was estimated with and without the tropospheric corrections.

Mitigation of tropospheric effects and the correct characterization of their spatial characteristics are critical in developing error models for missions that allow [5] or will allow [6] systematic interferometric processing on a global scale.

Figure 1 show a significant gain is attained when the tropospheric delay is corrected. As already pointed out in [1] the gain is region dependent. A very high gain is achieved where the model accuracy is also very good such as in Europe or the U.S. In only one of the 146 stacks, located in Indonesia, were the corrections found to slightly worsen the performance.

\section{ERROR-BARS VALIDATION}

Knowledge of displacement rate precision is of central importance in geophysical studies of large-scale tectonic movements where the precision requirements are often very high [7]. For the sake of completeness, the behavior of the displacement rate as described in Equation 4 must be verified in practice.This was achieved by cross-validation with GNSS measurements.

Given a full statistical characterization of the GNSS/InSAR differences [8][9], and assuming the GNSS accuracies to be correct, we can now verify if $\Gamma_{v}$ actually represents the variogram of the velocity error. First, from the vector $\underline{\Delta}$ of $N$ differences between GNSS and InSAR velocities, we compute all $\left(N^{2}-N\right) / 2$ unique pairwise differences between the elements. Their mean is expected to be zero, with $\sigma_{G, i}^{2}$ the error variance of the $i$-th GNSS measurement, their variance is

$$
\sigma_{\Delta}^{2}(i, j)=\mathbb{E}\left[\left(\Delta_{i}-\Delta_{j}\right)^{2}\right]=\sigma_{G, i}^{2}+\sigma_{G, j}^{2}+\Gamma_{v}\left(d_{i, j}\right)
$$

The scales at which $\Gamma_{v}$ can be verified by this method clearly depend on the spatial distribution of GNSS stations, with the range of scales being limited by the smallest and largest distances between stations. A statistic defined as the standardized difference

$$
T_{i, j}=\frac{\Delta_{i}-\Delta_{j}}{\sigma_{\Delta}(i, j)}
$$

should follow a standard normal distribution if the error description is correct. To test the validity of the variance model in Equation 5, a $\chi^{2}$-test can be performed on $\underline{T}$. The confidence region at significance level $\alpha$ for the true $\sigma_{T}^{2}$ is

$$
\sqrt{\frac{(N-1) \hat{\sigma_{T}^{2}}}{\chi_{1-\alpha / 2, N-1}^{2}}}<\sigma_{T}<\sqrt{\frac{(N-1) \hat{\sigma_{T}^{2}}}{\chi_{\alpha / 2, N-1}^{2}}}
$$

where the number of degrees of freedom is $N-1$ and $\hat{\sigma_{T}^{2}}$ is the sample variance of $T$. The validation approach will 
Expected Performance at $150 \mathrm{~km}$
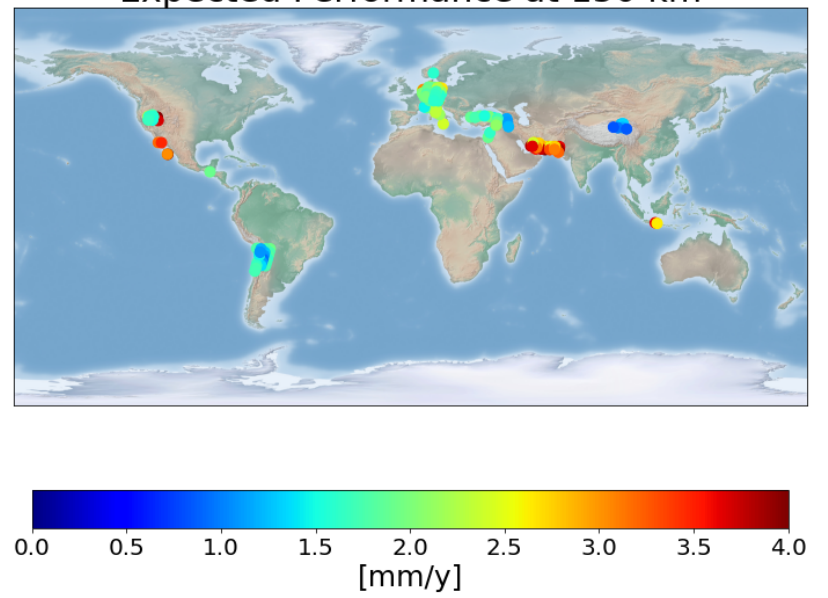

(a)
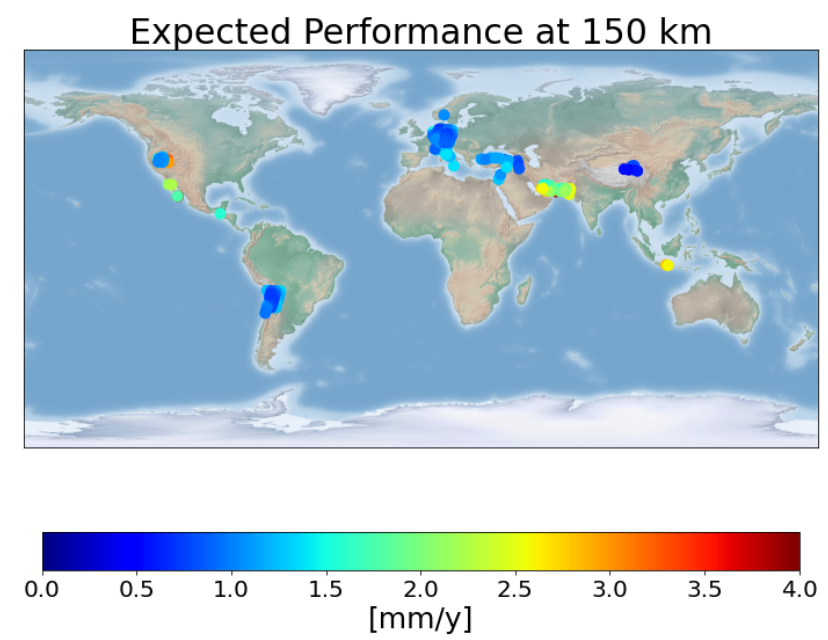

(b)

Fig. 1: Geographic distribution of the expected performance at $150 \mathrm{~km}$ : before (a) and after (b) the corrections..

focus on analyzing the distribution of $\underline{T}$ and the confidence interval for $\sigma_{T}$. The cross-validation scheme was performed on the German deformation map. The dataset includes 41 stacks acquired in both ascending and descending geometry. This region was chosen due to easy and open access to a very dense GNSS network from Nevada Geodetic Laboratories [10, 11] with all stacks containing sufficient GNSS stations.

The confidence intervals for $\sigma_{T}$ at $5 \%$ significance level are shown in Figure 2 (a). Of course, the dependence on the number of GNSS stations,is strong. However, for almost all stacks the confidence intervals include $\sigma_{T}=1$ indicating acceptance of the null hypothesis $H_{0}: \sigma_{T}=1$ versus the alternative $H_{1}: \sigma_{T} \neq 1$.

Figure 2 (b) shows the distribution of $\underline{T}$ pooled over all stacks, along with the nominal standard normal distribution under the null. The pooled value of $\hat{\sigma_{T}}$ is 1.03 , showing very good agreement between the model and the measurements.

\section{CONSIDERATIONS ABOUT THE DETECTION OF NON-LINEAR DEFORMATION}

To conclude it is worthwhile consider the capability of InSAR measuring the deviation of the time series from the model used for the temporal phase unwrapping. These signals can be very interesting for scientists and are often observed in tectonics motions (i.e. post-seismic deformation, slow slip events). While the achievable accuracy of the deformation rates takes advantage from a longer time series (Equation 4) this is not true when retrieving the non-linear part of the deformation signal that is bound to the accuracy of a single phase sample as indicated by $\bar{\Gamma}(d) / 2$ in Equation 4 . According to the data collected in this study, the accuracy of the single phase sample is on the order of centimeters (depending on the geographic area and time of day). The detectability of transient signals is determined by intensity and scale. Figure 3 shows the average of the variograms computed on short temporal baselines of the residual phase obtained compensating the linear model estimated with the temporal phase unwrapping. This should represent the accuracy in detecting transient signals at different scales. On scales grater than $100 \mathrm{~km}$, only several centimeters of non-linear deformations are detectable in InSAR time series while at medium scales $(40-50 \mathrm{~km})$ smaller event such like volcanic unrest are observable. Of course assumptions on the deformation models can improve this capability. Spatial/temporal filtering can improve the precision by trading it off against a reduced observability of the temporal/spatial scale of the deformation signal.

\section{REFERENCES}

[1] Xiaoying Cong, Ulrich Balss, Fernando Rodriguez Gonzalez, and Michael Eineder, "Mitigation of Tropospheric Delay in SAR and InSAR Using NWP Data: Its Validation and Application Examples," Remote Sensing, vol. 10, no. 10, 2018.

[2] H. Breit, T. Fritz, U. Balss, M. Lachaise, A. Niedermeier, and M. Vonavka, "TerraSAR-X SAR Processing and Products," IEEE Transactions on Geoscience and Remote Sensing, vol. 48, no. 2, pp. 727-740, 2010.

[3] F. R. Gonzalez, A. Parizzi, and R. Brcic, "Evaluating the impact of geodetic corrections on interferometric deformation measurements," in EUSAR 2018; 12th European Conference on Synthetic Aperture Radar, June 2018, pp. $1-5$.

[4] T. R. Emardson, M. Simons, and F. H. Webb, "Neutral atmospheric delay in interferometric synthetic aper- 


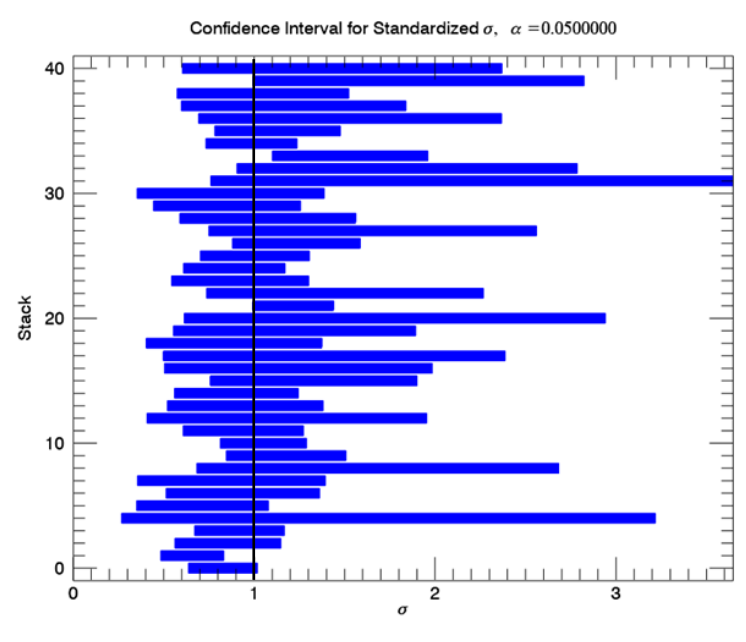

(a)

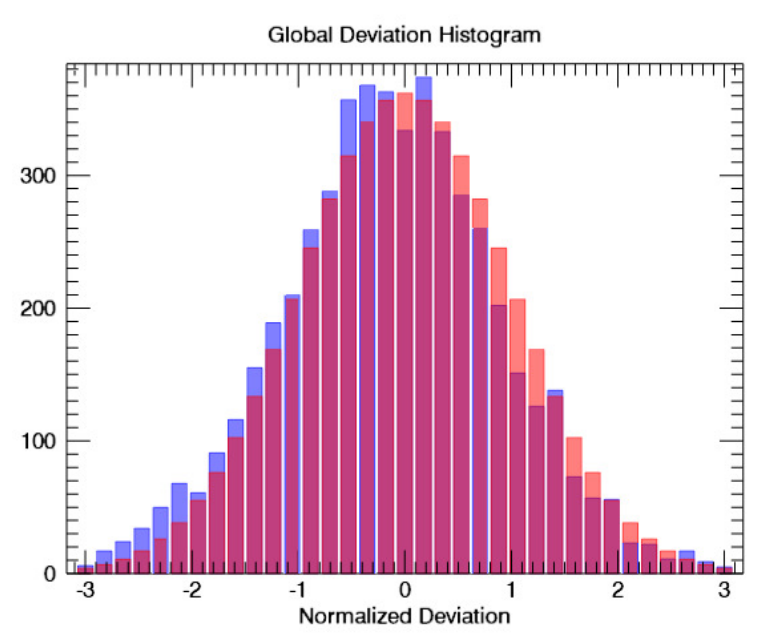

(b)

Fig. 2: Results of the InSAR/GNSS velocities cross-validation: (a) the confidence intervals where the black line denotes $\sigma_{T}=1$. (b) Histogram of the pooled vectors $\underline{T}$ using NGL GNSS results (blue) compared to the nominal $N(0,1)$ distribution (red).

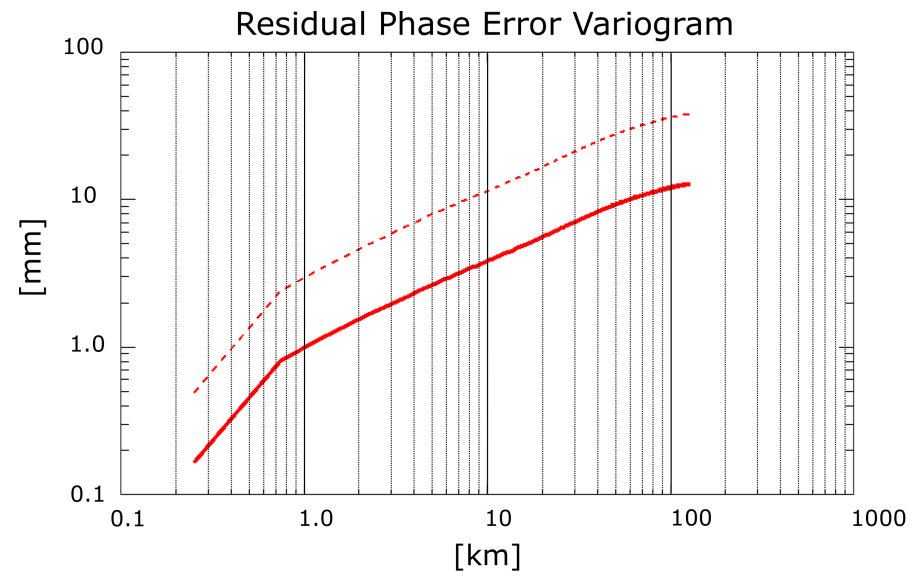

Fig. 3: Residual phase variogram $1 \sigma$ (bold line), $3 \sigma$ (dashed line)

ture radar applications: Statistical description and mitigation," Journal of Geophysical Research: Solid Earth, vol. 108, no. B5, 2003.

[5] Ramon Torres, Paul Snoeij, Dirk Geudtner, David Bibby, Malcolm Davidson, Evert Attema, Pierre Potin, BjÖrn Rommen, Nicolas Floury, Mike Brown, et al., "GMES Sentinel-1 mission," Remote Sensing of Environment, vol. 120, pp. 9-24, 2012.

[6] Paul A Rosen, Yunjin Kim, Raj Kumar, Tapan Misra, Rakesh Bhan, and V Raju Sagi, "Global persistent SAR sampling with the NASA-ISRO SAR (NISAR) mis- sion," in 2017 IEEE Radar Conference (RadarConf). IEEE, 2017, pp. 0410-0414.

[7] Tim J Wright, "The earthquake deformation cycle," Astronomy \& Geophysics, vol. 57, no. 4, pp. 4.20-4.26, 08 2016.

[8] A. Parizzi, F. Rodriguez Gonzalez, and R Brcic, "A Covariance-Based Approach to Merging InSAR and GNSS Displacement Rate Measurements," Remote Sens., vol. 13, no. 300, Jan. 2020.

[9] A. Parizzi, R. Brcic, and F. De Zan, "InSAR Performance for Large-Scale Deformation Measurement," IEEE Transactions on Geoscience and Remote Sensing, pp. 1-11, 2020.

[10] "MIDAS Velocity Fields. Available online: http://geodesy.unr.edu/ (accessed on 1 October 2018).,"

[11] Geoffrey Blewitt, Corné Kreemer, William C. Hammond, and Julien Gazeaux, "MIDAS robust trend estimator for accurate GPS station velocities without step detection," Journal of Geophysical Research: Solid Earth, vol. 121, no. 3, pp. 2054-2068, 2016. 